\title{
La motivación, herramienta pedagógica para impulsar el trabajo educativo y comunitario
}

\author{
Doris Miranda Moreno ${ }^{1}$
}

1 Egresada de la Maestría en Gestión del Desarrollo Comunitario. 1ra. Edición UNAN-Managua FAREM-Estelí. Correro electrónico: domimo85@yahoo.com

\section{RESUMEN}

La educación preescolar es la base del desarrollo afectivo, social, cognoscitivo y psicomotor que favorece el desarrollo integral de la niñez, en el municipio de Estelí. Para desarrollar este trabajo se cuenta con 185 educadoras voluntarias (95 urbanas, 90 rurales) llamadas Educadoras Comunitarias, que atienden a 2,678 niños y niñas que equivalen al $65 \%$ de la niñez atendida en el nivel preescolar en el municipio. Para ser Educadora es un requisito contar con educación primaria aprobada. Estas educadoras trabajan en los preescolares entre 3 y 4 horas, cinco días por semana y no reciben salario. Están organizadas en el Movimiento de Educadoras de Preescolares Comunitarios (MOVEDUPCE).

Esta investigación tiene como objetivo comprender las motivaciones individuales y colectivas que permiten que las educadoras comunitarias realicen una labor educativa integral con la niñez y la comunidad. Este estudio es de tipo cualitativo, se utilizaron métodos narrativos, como: grupos focales, y entrevistas abiertas. La investigación documental nos ubica en el contexto de la investigación y sus antecedentes históricos.

Entre los resultados más significativos se identificaron dos tipos de motivaciones que las impulsa a trabajar con responsabilidad y compromiso: a) Las motivaciones personales que se relacionan con su comportamiento maternal que les permite proveer de amor a los niños y niñas que llegan a la escuela y proteger sus derechos con alegría y felicidad; b) La motivación colectiva relacionada con su compromiso con la comunidad y el desarrollo. Estas motivaciones les permiten sentirse parte importante de la comunidad y elevar su autoestima y valoración personal. La labor de estas educadoras comunitarias ha trascendido su rol pedagógico porque han demostrado su capacidad para autogestionar proyectos, realizar coordinaciones interinstitucionales, asumir liderazgo, y promover la participación comunitaria en la organización y movilización social para la defensa de los derechos de las niñas y de las mujeres.

Palabras claves: pedagogía, trabajo educativo, trabajo comunitario, educadoras

\section{ABSTRACT}

Preschool education is the basis of affective, social, cognitive and psychomotor development, which promotes integral development of children, in the municipality of Estelí. To develop this research, has 185 volunteer educators (95 urban, 90 rural) called Educating Community, serving 2,678 children, equivalent to $65 \%$ of children treated at the preschool level in the municipality. To be Educator is a requirement to have passed primary school. These teachers work in the kindergarten around 3 and 4 hours, five days a week and they are not paid. They are organized in the Movement of Communitarians Preschool Educators (MOVEDUPCE). 
This research purpose is to understand the individual and collective motivations that permit to the community educators perform an integral educational work with children and the community. This study is qualitative, narrative methods were used, such as focus groups, and open interviews. The documental research places us in the context of the research and its historical background.

Among the most significant results two types of motivation were identified, which takes them to work with responsibility and commitment: a) personal motivations that relate to maternal behavior that allows them to provide love to the children who come to school and protect their rights with joy and happiness, b) collective motivation related to their commitment to the community and development. These motivations lead them to feel part of the community and increase their self-esteem and personal value. The work of these communitarians educators has transcended their pedagogical role because they have established their ability to self-manage projects, conduct interagency coordination, to assume leadership, and to promote community participation in the organization and social mobilization for the rights of children and women.

Keywords: pedagogy, educational work, community work, educators

\section{INTRODUCCIÓN}

El objetivo del presente estudio se centra en comprender las motivaciones individuales y colectivas que permiten que las educadoras comunitarias realicen una labor educativa integral con la niñez y la comunidad.

Dada la importancia de la educación preescolar, como base del desarrollo afectivo, social, cognoscitivo y psicomoto que favorece el desarrollo integral de la niñez, es importante visibilizar lo que impulsa a estas educadoras a realizar este trabajo educativo, no remunerado, con tanta responsabilidad y dedicación y que permite que estos niños y niñas se desarrollen. Atienden a unos 2,678 niños y niñas que equivalen al $65 \%$ de la niñez atendida en el nivel preescolar en el municipio de Estelí.

\section{MATERIAL Y MÉTODOS}

Se realizó una investigación cualitativa, porque es la que faculta hacer una aproximación a las situaciones sociales en las que están inmersas las educadoras comunitarias, para poder describirlas y comprenderlas.

La estrategia metodológica es cualitativa, naturalista, porque retoma principios del constructivismo e interpreta haciendo uso de Etnometodología, como perspectiva sociológica, que toma en cuenta los métodos que los seres humanos utilizan en su vida, para realizar acciones de la cotidianidad, tomar decisiones, establecer una conversación con los otros.

El área de incidencia de esta investigación cualitativa son barrios y comunidades del municipio de Estelí ubicado a $150 \mathrm{Km}$ al norte de Managua, nombrado como la cabecera departamental que lleva su mismo nombre, en la región norte de Nicaragua.

El municipio cuenta con 185 educadoras voluntarias (95 urbanas, 90 rurales) llamadas educadoras comunitarias, las que atienden directamente los preescolares, son mujeres, trabajan en los preescolares entre 3 y 4 horas, cinco días por semana. Es un requisito contar con educación primaria aprobada. Su labor no es remunerada y están organizadas en el Movimiento de Educadoras de Preescolares Comunitarios (MOVEDUPCE).

Los criterios de selección de la muestra no fueron criterios estadísticos, sino estructurales, con un grupo homogéneo que produce resultados muy simples y obvios. Se escogió a 20 educadoras que demostraron su voluntad para participar en la investigación. Se realizó discusión grupal la cual tenía el propósito 
de recrear la realidad lo más cercana posible, para que las educadoras expresen sus sentimientos y pensamientos. Se realizaron dos entrevistas abiertas con personas que ha estado inmersas en el trabajo educativo y comunitario de las educadoras.

\section{RESULTADOS}

Abordamos los hallazgos más significativos en el proceso de investigación los cuales se pueden resumir en:

\section{La motivación personal}

- Lo que más sobresale es el amor que tienen por los niños y niñas de su comunidad, lo que les provoca asumir la defensa de sus derechos.

- Seguir preparándose, para elevar su nivel educativo y académico. Este último aspecto, tiene relación estrecha con su necesidad de reconocimiento, valor y estima, que todo ser humano necesita y que permite también impulsar su crecimiento personal.

\section{Las motivaciones colectivas}

- El compromiso de servicio social con la comunidad, padres y madres, donde ellas son parte de la misma, donde han vivido siempre.

- Las educadoras comunitarias expresan de manera determinante, que su aporte es muy importante, porque con esto, ellas permiten que la niñez se eduque y que la comunidad poco a poco alcance mayores niveles de desarrollo para salir de la pobreza.

- Otro aspecto relevante es la práctica los conocimientos adquiridos durante el transcurso de su labor como educadoras, así mismo, la oportunidad de desarrollar nuevos conocimientos a partir de la puesta en práctica de nuevas metodologías aprendidas.
Estos resultados también cruzan con algunos factores que permiten realizar esta labor educativa y comunitaria Los factores sociales y políticos son los que prevalecen en relación con las motivaciones son los siguientes:

a) Factores políticos. Ser consideradas lideresas comunitarias, tanto por ellas mismas, como por los otros actores de la comunidad donde se insertan, les permite contar con el respeto de la misma comunidad, de ser ejemplo de vida para muchas mujeres.

b) Factores sociales La necesidad de apoyo a su comunidad para que esta se desarrolle, lo que permite también que se preparen para desempeñar su papel como guía para las madres y otros actores, es por esto mismo y por la confianza que la comunidad deposita en ellas, lo que las impulsa para mantenerse en esa labor social por mucho tiempo.

La labor de las educadoras comunitarias ha trascendido en su rol pedagógico de impartir clases a los niños y niñas de la comunidad donde viven, demostrando su capacidad para autogestionar propuestas de proyectos, realizar coordinaciones interinstitucionales, asumir liderazgo, promover participación comunitaria en la organización y movilización social para la defensa de los derechos de las niñas y también los derechos de las mujeres.

Esto permite que los preescolares comunitarios del municipio de Estelí cuenten con educadoras con gran motivación, ejerciendo su rol de lideresas, comunicándose con otros actores comunitarios de manera asertiva y dinámica y que se mantengan por muchos años al frente de los preescolares comunitarios. 


\section{RECOMENDACIONES}

Es necesario tomar en cuenta las siguientes recomendaciones para potenciar este trabajo educativo y comunitario que genera movilización alrededor de la educación y el desarrollo de cada barrio y comunidad.

1. Es un reto para las autoridades del municipio de Estelí, garantizar que se siga desarrollando el trabajo educativo desde los preescolares comunitarios, ya que es la vía de garantizar la educación preescolar en todos los barrios y comunidades del municipio.

2. El MINED, debe tener como un reto permanente la capacitación y profesionalización de todas las personas que asumen el reto de convertirse en educadoras comunitarias, para garantizar la calidad de los aprendizajes.

3. El Estado y las ONG deben promover la existencia del enfoque de la Educación Popular en todos los procesos que se desarrollen, promoviendo el respeto a comunidades y barrios, y su lógica de vida, sus intereses, necesidades, problemáticas y expectativas.

4. Todos los procesos emprendidos se deben trabajar bajo un enfoque de igualdad, sobre todo en el caso de las educadoras comunitarias, de las mujeres y de las personas con alguna discapacidad, o personas que por diversos motivos sufren mayor discriminación y menos oportunidades para un desarrollo integral. El enfoque de género es esencial, pues las mujeres son en su mayoría el motor de estas comunidades.

5. El importante que el estado, organizaciones civiles y la organización comunitaria, promueva el reconocimiento social sistemático, ya que este es un motor que garantiza la permanencia de las educadoras comunitarias, es importante destacar que para estas es fundamental, para reconocerse y valorarse en toda su dimensión.

6. Es muy importante para las educadoras comunitarias consolidarse en sus espacios organizativos, para garantizar la demanda de sus derechos, así mismo apoyar sus demandas para garantizar que se incremente el apoyo económico, pero que además, sea entregado en tiempo y forma por parte del MINED.

7. Se deben promover acciones de incidencia desde los espacios de coordinación de la sociedad civil, para el establecimiento de convenios con el MINED a fin de garantizar la estabilidad y políticas de estímulo a educadoras comunitarias.

8. Es importante que el MINED realice un trabajo sistemático de seguimiento a las estructuras comunitarias, llamados gabinetes de educación, en donde participan las educadoras comunitarias, a fin de acompañar la gestión de los mismos, esto permite ver la integralidad de la educación y la planificación de acciones más coherentes con la realidad de cada barrio y de las comunidades.

9. Se debe garantizar que los actores organizados en barrios y comunidades para defender el derecho de la educación, utilicen las herramientas técnicas con que cuentan, para dar seguimiento al trabajo educativo e informar al MINED de sus avances y dificultades, lo que permitirá retroalimentar el sistema de monitoreo, seguimiento y evaluación instalado a nivel del municipio. 


\section{BIBLIOGRAFÍA}

Álvarez Arzate, María Dolores; Rodríguez Rivera,

Naví Argentina (2010). Métodos de Investigación social Cualitativa. FAREM/CICAP, EstelíNicaragua, 93 pp.

Boedeker Francisco; Van de Velde, Herman. (2010), Metodologías y Técnicas de Comunicación Participativa. CURN-CICAP, Estelí - Nicaragua, 76pp.

Castillo Herrera, Beverly; Van de Velde, Herman (2010). Taller de Graduación. FAREM/CICAP, Estelí-Nicaragua, 61pp.

García, Nakord J. (1996) Organización Comunitaria-Algunas consideraciones necesarias, Managua-Nicaragua, 24pp.

lan, Gough. (2007/08) El enfoque de las capacidades de M. Nussbaum: Un análisis comparativo con nuestra teoría de las necesidades humanas, 9pp.

INSFOP (2007). Proyecto Movilización Social para la Educación Preescolar en el Municipio de Estelí. Presentado a Comisión Europea. PDV/2006/1567. Estelí, Nicaragua, $51 \mathrm{pp}$.

INSFOP y Casa del Tercer Mundo (2009). Evaluación intermedia del Proyecto "Movilización Social para la Educación Preescolar en el Municipio de Estelí". Evaluadora: María Erlinda Sandino. Estelí, Nicaragua, $36 \mathrm{pp}$.

Pérez M. y Van de Velde H. (2004). Procesamiento de Datos. CURN-CICAP, Estelí - Nicaragua, 68 pp.

Sáenz del Castillo, Andrés Ángel. 2008. Teoría Crítica de la Educación. Homepage Educritica. com 